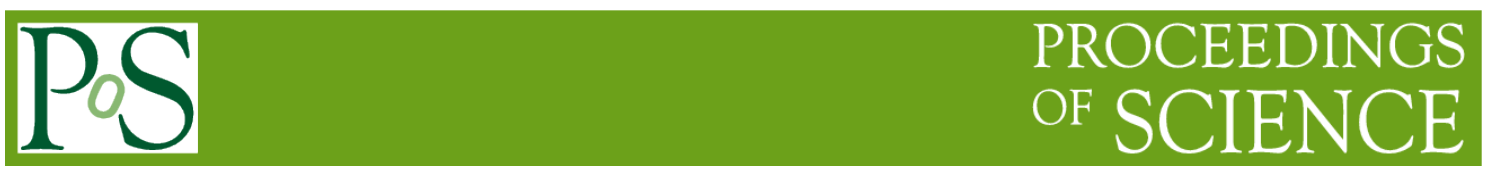

\title{
A Constraint-based Tourist Attraction Recommender System
}

\section{Yijin Shi ${ }^{1}$}

Tourism and Culture College, Yunnan University

Lijiang, Yunnan, China

E-mail: 349497232@qq.com

\section{Zhiyang $\mathrm{Jia}^{2}$}

Tourism and Culture College, Yunnan University

Lijiang, Yunnan, China

E-mail: jdyjazz@gmail.com

The paper develops a constraint-based tourist attraction recommender system. The recommended system acts as a web-based application. It can generate a recommendation list of attractions for the tourists. Traditional recommender systems, content-based filtering and collaborative filtering, for instance, would not be satisfied for the application of tourist recommendation. Tourist attraction recommendation can be deemed as a constraint satisfaction problem (CSP). The constraint-based recommender system is illustrated. In order to demonstrate the calculation process of the system, the case is described in detail. Some thought and systematically work in the future is also explored.

ISCC2017

16-17 December 2017

Guangzhou, China

\footnotetext{
${ }^{1}$ Speaker

${ }^{2}$ This study is supported by Scientific research plan projects of Yunnan Education Department (2016ZDX261) 


\section{Introduction}

Living in the Internet age, people find themselves more and more frequently confronted with the nasty problem of information overload. The recommender systems are deemed as the perfect solution to the problem by pushing daily choices for Internet users and helping them make decisions.

The classic method of developing recommender systems as extensively used in the IT industry is the famous collaborative filtering [1]. Simply speaking, CF algorithm is based on the hypothesis that interests groups have common experience.

The recommender system is seemed as a decision making algorithm for Internet users while it can push information that users may wanted. Also, the recommender system is considered as a tool of e-commerce that helps users find items or services which is computed according to users' interest, behavior history, preference and requirement etc [2].

The user-based collaborative filtering algorithm finds the users' preferences for goods or content (such as commodity purchase, collection, content comment or sharing) through the user's historical behavior data [3]. According to different users' attitude and preference to the same goods or contents, the relationship between the users is calculated as well as the merchandise recommendation between users with the same preferences. The basic idea of the item-based collaborative filtering algorithm is to calculate the score of the used item according to an user. It can predict the scores of unused items after having been used by the user, and then output them in descending order according to the predicted score. Items that have the highest score are the items that should be recommended to the user.

The case-based and constraint-based [4] are two wildly used knowledge-based recommender systems. The principle of the two knowledge-based algorithms is: the user's requirements are fristly recorded, and then the result of recommendation items are calculated. The distinct difference between them is the calculation process. The knowledge-based methods take the knowledge base into account.

With the continuous development of information technology, the consumption pattern of tourists has undergone a major change, the degree of dependence on information continues to increase [5], the application of network resources is also expanding, and the application and promotion of information recommendation technology is an important representative. The information recommendation technology helps tourists filter unnecessary information, and transmits the most effective information to tourists, which reduces the time to collect, collate, analyse of data and the cost of capital.

\section{Development of the Recommender System}

The constraint-based tourist attraction recommender system, as presented in this paper, can effectively integrate and diversify internal and external tourism resources to fulfill the users' requirements whenever they are confronted with the dilemma of information overload. The system provides logically calculated support in their decision-making process, ultimately recommending appropriate information of attractions for prospective tourists.

Components of the system include knowledge acquisition environment, GUI generation module, tourist attractions recommendation engine and a core knowledge base. Architecture of the recommender system is illustrated in Fig. 1. 


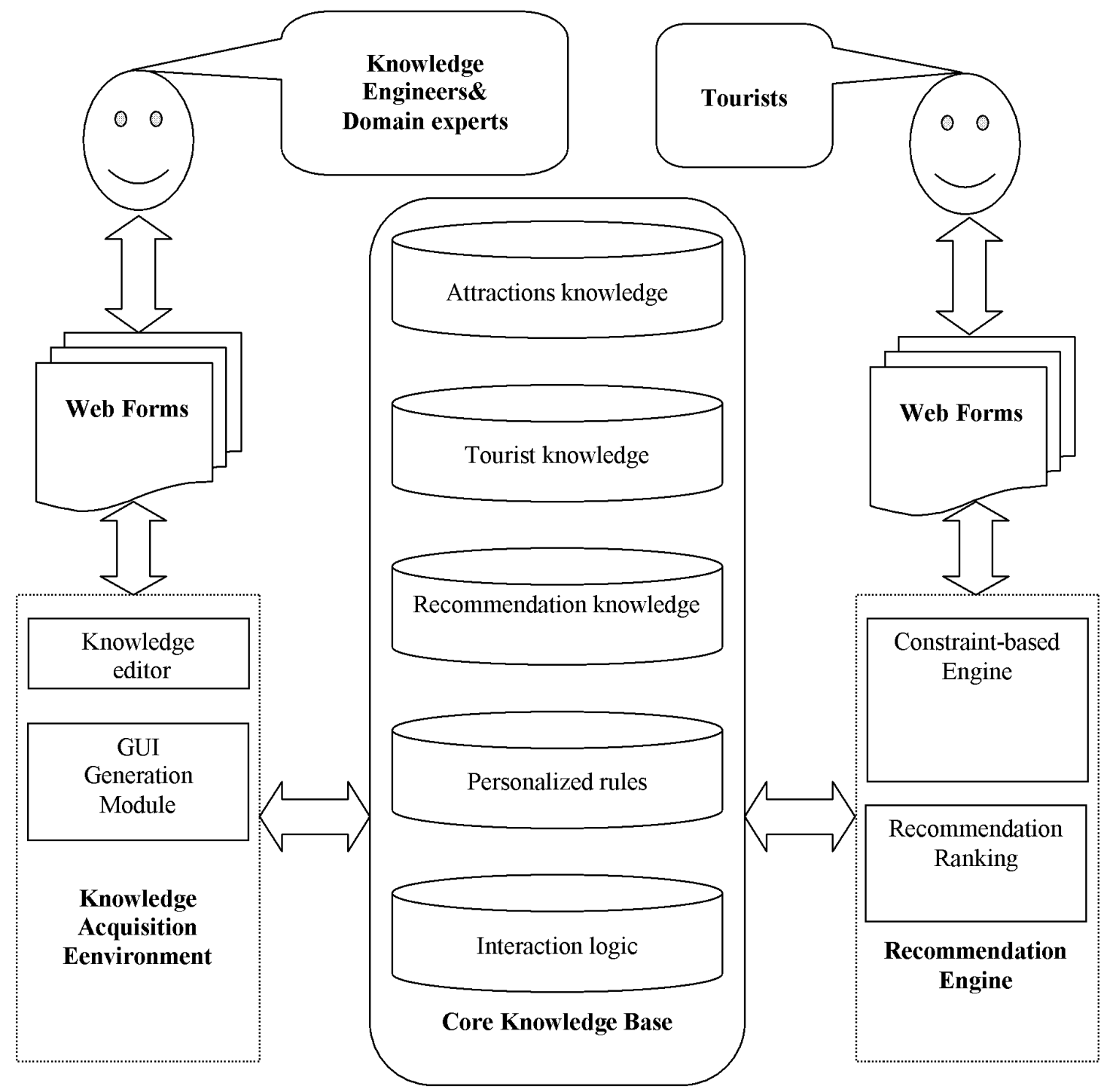

Figure 1 : The architecture of Constraint-based Tourist Attraction Recommender System

\subsection{Knowledge Acquisition Environment}

The knowledge acquisition environment provides several visualization tools of knowledge acquisition, which can help knowledge engineers and domain experts easily define and acquire domain knowledge, update and maintain the knowledge base.

The knowledge editing tools are used to design and maintain tourist attractions knowledge base, formal knowledge and store them to the core knowledge base. This knowledge includes the following content:

Attractions attributes that describe the structure of tourist attractions, such as service time of attractions, ticket prices, description of service standards and attraction category (natural landscapes, historical monuments or et al.) and other related tourism resources.

The tourists' attributes, which describe the preferences and requirements, such as the GUI dialog that may occur during the course of inquiring the interests and profile of tourists.

The amount of constraint blog visitors between attributes the amount of tourist attractions among several resource properties. These constraints contain the recommended rules of knowledge rules indicating personalization logic area. Constraints can be expressed in terms of the "If-Then" formula, such as "if a family that comes from urban takes a vacation, then the system can recommend some ancient town for the vacation." 


\subsection{GUI Generation Module}

The interaction process designer is used to construct interactive model and acquisitive knowledge interaction logic to help support personalized user session. The designer is composed of three kinds of user interface including session-independent customer profiles, static fill-out forms per-session and conversational recommendation dialogs

\section{Recommendation Engine}

Tourist attraction recommendation can be deemed as a constraint satisfaction problem (CSP) which can be descripted as a triple form [6]:

$V=\left\{V_{1}, \quad, V n\right\}$, where $V$ is a variables set,

$D=\left\{D_{1}, \quad, D_{n}\right\}$, where $D$ is a set consisting of specific domain values,

$C=\left\{C_{1}, \quad, C_{m}\right\}$, where $C$ is a set consisting of constraints.

$V_{i}$ can be assigned to the values in the domain $D_{i}$. Each constraint $C_{j}$ is such a pair $<t_{j}, R_{j}>$. And $t_{j}$ is a subset of $k$ variables. Furthermore, $R_{j}$ is a k-ary relation on the corresponding subset of domains $d_{j}$. A value of $v$ satisfies the constraint of $\left\langle t_{j}, R_{j}>\right.$ if the values were assigned to the variables $t_{j}$ satisfy the relation $R_{j}$.

For the tourist recommendation task, we can infer:

Here $V=\left\{V_{C}, V_{P}\right\}$, where $V_{C}$ is a set of variables according to the requirements of tourists, and $V_{p}$ is a set of features of tourist attraction in the base.

$D$ contains the domain definitions for variables in $V$.

$C=\left\{C_{P}, C_{I}, C_{F}, C_{O}\right\}, C_{P}$ is the constraint set of values taken from $V_{P}$, which includes all the attractions fitting tourists' wish; $C_{I}$ descripts the constraints (according to the tourist restrictions) set of values in $V_{C} ; C_{F}$ is a set consisting of constraints of the filters; $C_{O}$ is a set of other inconsistency constraints.

\subsection{Demo Case of Recommendation Calculation}

The requirements for attractions of tourist that can be represented by a set of variables as: $V_{T}=\{$ Maximum expense,Traveling preferences, Expected accommodation $\}$. According to this definition, Table 1 illustrates the requirements of a demo tourist.

\begin{tabular}{lll}
\hline Maximum expense & Traveling preferences & Expected accommodation \\
\hline $300 \mathrm{CNY}$ & Folk custom & medium \\
\hline
\end{tabular}

Table 1. Tourist Requirements Demo.

The properties of attractions can be represented by a set of variables as: $V_{P}=\{$ Attraction name,Total expense, Accommodation $\}$. The alternative recommendations are presented in Table 2.

\begin{tabular}{llll}
\hline Attraction name & Total expense & Accommodation & $\ldots$ \\
\hline Yulong snow mountain & $400 \mathrm{CNY}$ & None & $\ldots$ \\
Shuhe ancient town & $240 \mathrm{CNY}$ & holiday inn & $\ldots$ \\
The old town of Lijiang & $350 \mathrm{CNY}$ & holiday inn & $\ldots$ \\
$\ldots$ & $\ldots$ & $\ldots$ & $\ldots$ \\
\hline
\end{tabular}

Table 2. Tourism Attractions List.

The filters and the corresponding constraints set $C_{F}$ are demonstrated in Table 3. These constraints describe when a particular tourist attraction should be recommended.

\begin{tabular}{lll}
\hline Filters & Criteria & Conclusion \\
\hline \multirow{2}{*}{ Filter 1 } & Traveling preferences=Folk custom & $\begin{array}{l}\text { Shuhe ancient town and the old } \\
\text { town of Lijiang are Folk }\end{array}$
\end{tabular}


custom

The accommodation is

required to be better (equal)

Filter 2 Expected accommodation=Medium than three-star hotels and to be less (equal) than four-star hotels and the kind need to be holiday inn.

Table 3. Filters and Constraints List

Table 4 describes some inconsistency constraints, including the combinations which are impossible to be achieved, as contained in the Set $C_{O}$.

\begin{tabular}{ll}
\hline ID & Constraint \\
\hline Constraint 1 & Expected accommodation=High Or (Maximum expense $=<500)$ \\
Constraint 2 & Travelling preferences $=$ Folk custom Or (transport facilities $=$ good)
\end{tabular}

Table 4. Inconsistency Constraints List

For a given task of tourist attraction recommendation, the ideal solution is to calculate the complete valuation with the following set of $\left\{\mathrm{C}_{1}, \ldots, \mathrm{C}_{m}\right\}$. As a result, the tourist attraction meets the requirement of Table 1 and other constraints is the "Shuhe ancient town".

\section{Conclusions and Future Work}

In this paper, we discuss how to develop a constraint-based tourist attraction recommender system. Also, we can identify several challenges for the system applications [7]. Some topics will be explored in the future. A constraint-based recommendation mainly relies on its knowledge base [8]. It needs to build the knowledge base correctly and completely so as to guarantee worthwhile recommendations. As a result, the maintenance tasks of the system is challenging especially for the tourism service.

\section{References}

[1] Paolo Cremonesi, Franca Garzotto, Sara Negro et al.. Comparative evaluation of recommender system quality. CHI '11 Extended Abstracts on Human Factors in Computing Systems,2011: 19271932.

[2] Gülfem Işıklar Alptekin, Gülçin Büyüközkan. An integrated case-based reasoning and MCDM system for Web based tourism destination planning. Expert Systems with Applications. 2011,38(3): 2125-2132.

[3] Xiaoyuan Su, Taghi M. Khoshgoftaar. A survey of collaborative filtering techniques. Advances in Artificial Intelligence. 2009,2009(January):1-19.

[4] Adomavicius, G., Tuzhilin, A.. Toward the next generation of recommender systems: a survey of the state-of-the-art and possible extensions. IEEE Transactions on Knowledge and Data Engineering, 2005,17(6): 734-749.

[5] Lu, E.H.-C., Chih-Yuan Lin, Tseng, V.S.. Trip-Mine: An Efficient Trip Planning Approach with Travel Time Constraints. 2011 12th IEEE International Conference on Mobile Data Management (MDM). 2007: 152-161.

[6] Makoto Yokoo. Constraint Satisfaction Problem. Distributed Constraint Satisfaction, Springer Series on Agent Technology, 2001:1-45.

[7] Dietmar Jannach, Markus Zanker, Matthias Fuchs. Constraint-based recommendation in tourism: A multi-perspective case study, Information Technology \& Tourism. 2009, 11(2):139-155.

[8] A. Felfernig, G. Friedrich, D. Jannach, M. Zanker. Developing Constraint-based Recommenders, Recommender Systems Handbook, 2011:187-216. 